\title{
Descentralización y financiamiento de los municipios en Venezuela
}

\section{Brito, Dionisio*}

\section{Resumen}

EIMunicipio en Venezuela que aparecía relegado en el proceso político-administrativo ha adquirido en el proyecto descentralizador especial relevancia, a partir de la Reforma del Estado iniciada a fines de los años ochenta. El propósito del siguiente trabajo es abordar desde la perspectiva de la ciencia política los mecanismos y formas de relacionamiento del gobierno local con los entes del Gobierno Central encargado de administrar programas de asistencia financiera y técnica a los municipios. Partimos del presupuesto de que los fondos y programas elaborados en el nivel central del gobierno persiguen el relanzamiento del sistema político. En tal sentido se resaltan los aspectos relevantes de cada programa, tanto su normativa como sus implicaciones políticas. Se concluye con una reflexión a cerca del control que ejerce el Ejecutivo Nacional sobre los gobiernos locales a través de la intermediación que ejerce con base en las orientaciones de los organismos multilaterales que apoyan el proceso descentralizador en el ámbito municipal y su concepción de la vida local.

Palabras clave: Descentralización, Estado, multilaterales, municipios, programas de financiamiento, Venezuela.

\footnotetext{
Recibido: 98-10-06. Aceptado: 99-09-29
}

Lic. en Educación -Area Historia-. Maestría en Ciencia Política. Candidato a Doctor en Ciencia Política de LUZ. Profesor Asociado a Dedicación Exclusiva y Jefe de la Cátedra Socio-Politica Venezolana en el Programa Educación de la Universidad Nacional Experimental Rafael Maria Baralt. Cabimas Venezuela. Tlef 064-715644. Fax 064-415309. 


\section{The De-Centralization and Financing of Municipalities in Venezuela}

\section{Abstract}

The municipality in Venezuela that seemed to be exiled from the political and administrative process, has acquired special relevance in the de-centralization project since the beginning of state reform initiated at the end of the $1980 \mathrm{~s}$. The purpose of this paper is to approach, from a political science perspective, the mechanisms and relational aspects of local governments with the agencies of the central government that are in charge of administering programs of financial and technical assistance to said municipal governments. This is done from the perspective that funds and programs elaborated by the central government look to re-launch the political system. In this vein, the relevant aspects of each program are pointed out, both from a normative point of view, and with a view to the political implications. The conclusion is a reflection on the control exercised by the National Executive Branch on local governments through the exercise of its mediation based on the orientation of multilateral organisms that support the de-centralization process at the municipal level and on its conception of local life.

Key words: De-centralization, States, multilateral organisms, municipalities, financial programs, Venezuela.

\section{Introducción}

Asistimos a un momento de cambio sobre el papel del Estado, es ya un lugar común que en distintos foros y diversos trabajos, nos indiquen que el Estado actual necesita de modificaciones en el corto plazo, ello es elocuente. Por esa razón es frecuente encontrar términos como reestructurar, rediseñar, repensar nuevos modelos, ect., etc., en diferentes trabajos sobre esta cuestión.

En cuanto al crecimiento del número de Municipios en la estructura juridico-politica del Estado venezolano, tenemos que para 1989 Venezuela tenia 267 Municipios, hasta 1998 las Asambleas Legislativas respectivas han creado 330 Municipios, por cuanto existe una evidente demanda de los ciudadanos que aspiran el elevar a la categoria de Municipio, las parroquias en donde habitan; además algunos de estos Municipios también han sido creados a partir de cálculos partidista en atención a sus propios intereses electorales.

En este orden de ideas abordaremos el nuevo tipo de relación entre dos niveles del Es* tado, vale decir, la relación entre el Poder Ejecutivo y el Municipio en un contexto globalizado de alcance mundial. Esta realidad producto del proceso de descentralización iniciado en 1989 con un conjunto de decisiones politicas y normativas, constituye un objeto de estudio de la Ciencia Política, he alli su pertinencia. En definitiva el proceso de descentralización y sus implicaciones son básicamente una oferta política que procura atender una demanda que proviene de la sociedad civil pero impulsada y promovida desde el mismo Estado. En tal sentido, el objetivo del siguiente trabajo es 
estudiar los mecanismos por los cuales el Estado, por intermedio del poder central, controla las formas de financiamiento a los gobiernos locales utilizando fondos y programas, lo que nos remite, necesariamente, a la función del gobiemo local en el sistema político venezolano, en tal sentido se han implementado modelos que buscan suministrar mayor eficiencia a la gestión local en el marco del proceso descentralizador.

Sin duda que temas como reestructuración del Estado, el nuevo rol de la administración publica, descentralización, gobernabilidad y forma de participación son aspectos cardinales del debate político venezolano de finales del siglo $X X$.

\section{Estado y descentralización en Venezuela}

El Estado Venezolano se ha caracterizado en los últimos años por una acelerada pérdida de legitimidad, parece evidente que le resulta dificil cumplir sus obligaciones con la sociedad tanto en el plano del bienestar material como el espiritual. Un Estado deslegitima. do por los sucesos políticos y sociales del $27 \mathrm{y}$ 28 de Febrero de 1989 y los intentos de golpes de Estado del 04 de Febrero y 27 de Noviembre de 1992 y la sucesiva abstención electoral, tiene indefectiblemente efectos en el sistema político venezolano instaurado a partir del modelo diseñado en 1958; lo que Mirian Komblith denomina "Crisis del Sistema Populista de Conciliación de Elites y Crisis del Modelo Rentista". Estas evidencias empíricas y sus respectivas interpretaciones desde la óptica de la ciencia política son coincidentes con los trabajos de Carlos Blanco que al referirse al proceso de descentralización, precisa..... "La orientación fundamental del proceso de reformas fue la de promover el fortalecimiento del Estado y la necesidad de superar a un Estado débil, incapaz de asumir adecuadamente las funciones de su irrenunciable competencia. La experiencia demostró en los procesos criticos de 1992, cuando el ga no central perdió una alta dosis de apoyo y legitimidad social, que los representantes electos en estados y municipios podian ser factores de consenso y estabilidad institucional" ( Blanco, 1997: 17).

Ciertamente a partir de la década de los noventa la descentralización pasa a ocupar un lugar preponderante en el discurso político venezolano y las propuestas elaboradas en la Comisión Presidencial Para La Reforma Del Estado - COPRE - desde 1985 fueron aceleradas y muchas de ellas convertidas en leyes de la República. Esta primera consideración nos permite establecer que la descentralización es un hecho de naturaleza política, no solamente porque proviene del Estado y de la administración pública, que es en si misma un tenómeno político, sino también por sus efectos en el sistema político. En segundo lugar, conviene resaltar que la descentralización no es la única consecuencia del proceso de reforma del Estado. Dicha reforma tiene muchas aristas, y en tal sentido Nuria Cunill afirma: "La reforma del Estado se convierte en un tema clave a partir de segunda mitad de la década de los ochenta. Las agendas, aunque variadas, ofrecen dos prototipos. Uno, que pone el acento tanto en la democratización como en la modernización del Estado, y otro, que prioriza la reforma económica. El primero, aunque relativamente fallido, no por casualidad se desarrolla en Venezuela, país que en la época aludida aun no experimentaba los efectos de la crisis fiscal ya que mantenia niveles aceptables de renta petrolera " (Cunill, 1997:209).

En consecuencia, al Estado venezolano en la actualidad no sólo lo afecta una crisis de legitimidad ya señalada, sino también una crisis fiscal. Se necesitan fondos y programas como el Fondo Intergubernamental para la Descentralización( FIDES), el Fondo de Inversión Social de Venezuela (FONVIS) y el Proyecto de Mejoramiento Urbano en Barrios (PROMUEBA).

La renta petrolera ya no puede sostener el modelo de administración burocrático - populista asociado a dicha renta petrolera (Ochoa et al, 1996) por lo tanto es necesario ir 
a una reestructuración del Estado venezolano. En esta nueva realidad, Ochoa (1998.5), sostiene": Si bien es cierto que las propuestas neoliberales más radicales le asignan al Estado un rol pasivo en la economia, la práctica ha demostrado, que al menos en los países subdesarrollados el Estado tiene una participación activa en el proceso de reestructuración económica en el contexto neoliberal".

Las anteriores consideraciones teóricas nos permite elaborar la siguiente formulación hipotética: El Estado venezolano ha elaborado fondos y programas como el FIDES, FONVIS Y PROMUEBA a los fines de apoyar el proceso descentralizador en el área municipal, con el objetivo de relegitimar el sistema político.

El anterior enunciado nos lleva a establecer una correlación entre descentralización y Gobernabilidad, de lo cual nos ocuparemos en el desarrollo del artículo, abordando en el análisis tanto la perspectiva política como el componente económico, que a la luz de las nuevas realidades necesita una reestructuración del Estado acorde con los nuevos desarrollos del mundo capitalista contemporáneo, por esa razón encontramos en estos procesos una muy activa presencia de la banca y organismos multilaterales, tal y como lo veremos a continuación.

Los convenios de gestión establecidos entre el municipio y los organismos señalados, son la modalidad implementada para crear el nuevo paradigma y el nuevo tipo de cultura estatal en la administración publica, en la búsqueda de eficlencia y de nuevos modelos de legitimación de la acción del Estado, en el intento de ruptura del anterior modelo burocrático-populista y la implantación de los nuevos modelos de gerencia pública, claramente signados por las directrices de los organismos multilaterales, construyendo viabilidad politica al proceso de descentralización, tal y como lo señala Ochoa (1998:16) "Las pautas que orientan el proceso de modernización administrativa de los Estados en América Latina, las suministran los organismos multi- laterales, suasesoría es fundamentaimente de tipo sectorial, para lo cual han desarrollado propuestas especificas: Educación, Salud, Tributaria, etc., aunque con orientaciones generales sustentadas en la propuesta del llamado modelo de gerencia pública“.

La afirmación anterior nos posibllita hacer dos comentarios. En primer lugar, el proceso de modernización de la gestión publica es hoy un hecho en Latinoamérica, confirmado por la experiencia de paises vecinos registrados en diversos trabajos. En segundo lugar, permite subrayar la relación Estado-Democracia y Descentralización no sólo en la instancia municipal sino también en el plano regional (léase Gobernación). Sobre ello se han producido varias experiencias en la América latina. Carlos De Mattos (1990) es de la opinión que la idea de la descentralización como medio para promover el desarrollo local no es nueva.

En el diseño del nuevo modelo se ha producido también una nueva forma de rela* cionamiento entre el poder central y los gobiernos locales, atendiendo un nuevo tipo de racionalidad instrumental y económica, controlado por el poder central por medio de la via administrativa. En el plano politico administrativo el trabajo de Cristian Von Haldenwang (1990:62), da cuenta de este proceso al señalar: "La descentralización administrativa apunta al aumento de la eficiencia en la asignación y apropiación de Recursos por parte de las administraciones nacionales.

\section{Hacia un nuevo modelo de gestión municipal}

En el plano descriptivo debemos señalar que los fondos y programas son administrados por el poder central (léase Poder Ejeçutivo Nacional). A pesar que tienen la misma direccionalidad de apoyo a la gestión local, tienen sus especificidades desde el punto de vista formal, a saber:

ELFIDES tiene como marco normativo la Ley que lo creó en Enero de 1997 en atención a lo establecido en la Ley Orgánica de 
Descentralización, Delimitación $Y$ Transferencia de Competencias Del Sector Público. Adscrito el mencionado fondo al Ministerio de Relaciones Interiores, es financiado en un porcentaje del $15 \%$ al $20 \%$ de

Recursos provenientes del impuesto al consumo suntuario y ventas al mayor, transformado en el año 1999 en impuesto al valor agregado (IVA). En el artículo 12 de la ley que cré el FIDES, puede leerse: "EI Fondo Intergubernamental para la descentralización tendrá por objeto apoyar administrativa y financieramente el proceso de descentralización, atendiendo a criterio de eficiencia, compensación territorial, y de desarrollo regional, a tal afecto tendrá como funciones principales:

1. Determinar, asignar y administrar tanto global como individualmente, la participación de los Estados y Municipios en los re. cursos del fondo.

2. - Identificar, negociar y canalizar hacia los Estados y Municipios recursos financieros destinados a impulsar la descentralización $y$ el desarrollo regional" (FIDES, 1997:12).

EI FONVIS es una fundación adscrita al Ministerio de la Familla, formalmente creada en 1990 para mejorar las condiciones de vida de las comunidades, enfrentar la pobreza, consolidar la modernización del sector social y contribuir con el proceso de descentralización del Estado. Inclusive tiene un programa especifico a nivel de gobiemos municipales y administrados por el Programa de Naciones Unidas para el Desarrollo - PNUD-, para la inversión en infraestructura de servicios y fortalecimiento institucional denominado PROINSOL (Programa De Inversión Social Local). La misión del FONVIS es "Financiamiento de Proyectos de Servicios Públicos en Infraestructura; Fortalecimiento Institucional para los gobiernos regionales, locales y organizaciones civiles, mediante metodologías diseñadas para incidir positivamente en la eficiencia del gasto en los procesos de inversión" (FONVIS, 1998: 3).
EI PROMUEBA está adscrito a la Fundación para el Desarrollo de la Comunidad (FUNDACOMUN) y al Ministerio de Desarrollo Urbano, creado formalmente para enfrentar la pobreza crítica como una vía para contribuir con los gobiernos locales en el co-financiamiento, ejecución e inversiones en infraestructura urbana y equipos de aseo urbano, en obras como: Redes locales de distribución de aguas potables, redes locales de alcantariliado (cloacas), redes locales de distribución de energia eléctrica, redes locales de drenajes superficiales (brocal - cuneta), vias locales de acceso y vías internas peatonales de los barrios y adquisición de equipos de aseo urbano y domiciliario. Según FUNDACOMUN (1993: 3): "El Proyecto de Mejoramiento Urbano en Barrios -PROMUEBA-, es el primero en su tipo que se realiza en Venezuela. Para ello, se aprovecha la experiencia de FUNDACOMUN, a nivel nacional, y del Banco Mundial en proyectos desarrollados en Africa, Asia y en paises de América Latina. EI PROMUEBA consiste en la construcción de obras de infraestructura y la adquisición de bienes, destinados a mejorar las condiciones de vida de las poblaciones mas necesitadas, asentadas en los barrios pobres de las principales ciudades del pals e igualmente el PROMUEBA comprende el fortalecimiento de los municipios a través de la asistencia técnica y reforzamiento de FUNDACOMUN para que cumpla mejor con sus objetivos".

A pesar de su especificidad, los programas anteriormente señalados tienen varios elemenits comunes que presionan hacia cambios en la gestión municipal.

En primer lugar están vinculados de alguna manera a los entes multilaterales, bien sea a través del financiamiento directo o mediante la utilización de metodología y de control de los resultados, aplicando al sector püblico criterios de la empresa privada.

En segundo lugar, aspiran darle soporte financiero y técnico al proceso descentrali- 
zador en el ámbito municipal ante la debilidad de las tinanzas publicas municipales, pero manteniendo centralizadas las decisiones fundamentales en el modelo organizativo descrito $y$ establecido en su normativa vigente.

En tercer lugar, los modelos de gestión del FIDES, FONVIS y PROMUEBA, comportan un nuevo tipo de relacionamiento entre el poder central y el municipio atendiendo a una racionalidad económica cualitativamente diferente a los mecanismos tradicionales de intermediación que ejercen los partidos políticos, se trata según este modelo de gestión, de suministrar eficiencia a la gerencia pública local para enfrentar exitosamente las nuevas realidades regionales y municipales.

En cuarto lugar, estos programas exigen a los municipios, funcionarios capacitados tanto en la elaboración de los proyectos de obra de infraestructura como en el cálculo de las valuaciones para el pago a tiempo a las empresas contratistas ejecutoras de las obras. Gustavo León (1996) en trabajo sobre la descentralización realizado para le FIDES, plantea que el Estado no solamente debe atender la insatisfacción ciudadana, sino también promover la eficiencia en el gasto de la administración pública. En términos generales a dichas instituciones se les exigen la profesionalización del recurso humano al servicio de la administración pública y la formación del funcionario con cursos altamente especializados en gerencla pública, 10 cual es además un elemento favorecedor de la eficiencia.

\section{Condicionantes del proceso de decisiones}

Hasta el presente el proceso de descentralización ha tenido diversas implicaciones tanto en el plano del liderazgo politico como en la promoción de la participación, bien sea en la participación cludadana tradicionalmente conocida, como por intermedio de las organizaciones no gubernamentales, to que (Cunill, 1997) llama "La ampliación del espacio público".
A pesar que la participación en sus diferentes manifestaciones no es el objeto de estudio de este trabajo, estamos obligado a registrarlo como un hecho notorio del proceso de descentralización, los gobiernos locales participan en el directorio de organismos como el FIDES a través de dos representantes y sus respectivos suplentes, postulados por la Asociación Nacional de Alcaldes; y correlativamente según sea el ámbito regionalo municipal son los gobernadores o alcaldes, los responsables de evaluar las necesidades, definir prioridades, y elaborar los proyectos en su entidad respectiva. Para los efectos de los gobiemos locales la participación de los Alcaldes está garantizada, de acuerdo a lo establecido en la ley que creó el FIDES, no es este el caso del FONVIS y eI PROMUEBA que corresponden a una iniciativa del poder ejecutivo nacional.

Formalmente las solicitudes y/o proyectos son presentados por los Alcaldes en atención a indicadores sociales y evaluados de acuerdo a la satisfacción de necesidades básicas de la población y equidad en la distribución de los recursos por parte de los departamentos técnicos de los organismos ya mencionados, el FIDES, el FONVIS, y el PROMUEBA, pero en la practica el mecanismo por medio del cual se elige al presidente de la Asociación Nacional de Alcaldes, con una fuerte influencia partidista, condicionan el proceso, con las implicaciones políticas que ello comporta en las decisiones. En definitiva, en este contexto la mayoría de los casos el proceso concluye en una negociación entre el organismo nacional (FIDES, FONVIS O PROMUEBA) y el Alcalde respectivo. Burlaud y Laufer (1989:279) sostienen que: "Esta tendencia a la negociación se encuentra en la práctica cotidiana de la administración aunque no este escrita en la ley".

Lo que indica que el proceso no es mecánico, el mismo comprende una etapa de negociación y el reconocimiento a limitaciones politicas y técnicas presentes en las instancias de tomas de decisiones que lesionan la autonomia municipal, a pesar que todos los discur- 
sos son coincidentes en la necesidad de ampliar la capacidad de gestión y los espacios de políticas públicas de los gobiernos locales.

En buena medida este tipo de modelo parte de la premisa que a los gobiernos locales se le deben delegar funciones, elevando sus niveles de eficiencia, especialmente en la prestación de servicios públicos y servicios sociales. Esta corriente de pensamiento podemos ubicarla en la tradición anglosajona, que supone un gobierno municipal tutelado por el poder central. Todo ello obliga a los gobiernos locales a motivar y desarrollar una nueva cultura local, por cuanto el mencionado modelo le asigna a los gobiernos municipales y a sus unidades, la responsabilidad de ejecutar las politicas previamente diseñadas, atendiendo eficientemente las demandas ciudadanas.

Los lineamientos que el nuevo gobierno nacional elabore para el ámbito municipal permitirá hacer nuevas consideraciones sobre la evaluación de los problemas, el proceso de negociación, la toma de decisiones y la implementación y ejecución de las políticas públicas locales.

Conviene recordar que el Municipio juega un papel estelar en el proyecto descentralizador, el proceso de reforma del Estado iniciado en 1989 incluye a gobernaciones y alcaldias, ha planteado la necesidad de repolitizar el debate acerca de la orientación de la administración pública como ente estatal. En tal sentido hemos realizado una aproximación de carácter teórico. Resulta prematuro hacer un balance definitivo de un proceso aún en marcha, sin embargo podemos afirmar que ante la debilidad financiera de los municipios en Venezuela, programas como el FIDES, FONVIS $Y$ PROMUEBA han contribuido a mejorar la capacidad de gestión de los gobiernos locales de acuerdos a numerosas referencias empiricas, en obras de infraestructura, servicios y programas sociales, que han dotado al Estado Venezolano de cierta capacidad de gobernabilidad en un escenario de crisis económica y fiscal.

\section{Conclusiones}

El proceso de reforma del Estado actualmente en curso, no se ha desarrollado exclusivamente en Venezuela. Existen en tal sentido diferentes puntos de vistas y propuestas de modernización; empero ello no es sólo un problema técnico-administrativo de búsqueda de eficiencia y eficacia, es también un problema político en cuanto se refiere a la reestructuración del Estado Venezolano; yel rediseño de éste que responda al nuevo modelo económico cuyo eje central es la promoción de la economía del mercado. Dicho modelo necesita la presencia de un Estado en correspondencia con sus objetivos e intereses, por esa razón términos como flexibilización, competitividad, productividad son hoy componentes frecuentes en el discurso político-económico del presente. La estrategia modernizadora implica privatización, desburocratización y descentralización.

En este contexto se desarrolló nuestra hipótesis, que partió de un nuevo tipo de relación entre el poder central y el municipio, en el contexto del nuevo modelo de gerencia pública que se intenta construir. En el nuevo modelo el Municipio juega un rol relegitimador del Estado, atendiendo obras de infraestructura, equipamiento físico, servicios públicos y atenuando los conflictos de pobreza critica; Claramente influenciado por los organismos multilaterales, que le asignan al gobierno local funciones administrativas y reguladas por el poder ejecutivo nacional. Ahora bien, los alcances de los programas de los organismos multilaterales no se limitan al ámbito local, tienen otras expresiones en otros niveles sub nacionales y del Estado en general.

Adicionaimente el trabajo genera la posibilidad de retomar el debate en torno a la dicotomia política-administración, revisar el viejo modelo y evaluar el nuevo paradigma en el nivel municipal, a la luz de los recientes desarrollo teóricos, de una discusión que no se agota con la finalización del presente siglo. 


\section{Bibliografía citada}

Blanco, Carlos (1997). "El Proceso De Descentralización En Venezuela. Análisis crítico": Documento del BID. Washington. USA.

Burlaud, Alain y Laufer, Romain (1989). Dirección Publica: Gestión y Legitimidad INAP. Madrid. España.

C.O.P.R.E.O.E.A. (1998). Participación Ciudadana y Democracia. Editorial Texto. Caracas. Venezuela.

Cunill Grau, Nuria (1997). Repensando lo Publico a Través de la Sociedad. CLAD y Nueva Sociedad. Caracas. Venezuela.

De Mattos, Carlos (1990). "La Descentralización ¿Una nueva panacea para Impulsar el desarrollo Local?" En: Economia y Sociedad No. 3. Revista de la Universidad Católica de Chile. Santiam go. Chile.

FIDES (1997). Marco Legal. Ministerio de Relaciones Interiores. Caracas. Venezuela.

FONVIS (1998). Recurso para ef Desarrollo. Ministerios de la Familia. Caracas. Venezuela.

FUNDACOMUN (1993). Instructivo para la Participación de los Municipios en el Proyecto de Mejoramiento Urbano en los Barrios -Promueba -, Caracas. Venezuela.

Haldenwarg, Christian (1990). "Hacia un Concepto Politologico de la descentralización del Estado en América Latina" En: Eure. N.- 50.61-77. Instituto de Estudios Urbanos. Santiago. Chile.

Komblith, Miriam (1994). "Crisis Politica, Nuevas Reglas y Estabilldad de la Democracia en Venezuela". En: Politeia No. 17. Institutos de Estudios Politicos. Facultad de Clencias Juridicas y Políticas. UCV. Caracas. Venezuela.

León Villalba, Gustavo (1996). Descentralización en Venezuela. FIDES. Ministerio de Relaciones interiores. Caracas. Venezuela.

Ochoa Henríquez, Haydẻe. López Valladares, Mitha y Rodríguez, Isabel (1996). "Administración Pública y Populismo en Venezuela", En: Revista Venezolana de Gerencia. Vol., 1 No, 1. Vicerectorado Académico de la Universidad del Zulia. Maracaibo. Venezuela.

Ochoa Henriquez, Haydée (1998). "Reflexiones Teóricas para el Estudio de la Modemización Administrativa de las Gobernaciones en Venezuela". (Mimeo). Documento del Centro de Estudios de la Empresa. Facultad de Ciencias Económicas y Sociales. Universidad del Zulia. Maracaibo. Venezuela. 